\title{
Low-dose radiation therapy (LDRT) for COVID-19 and its deadlier variants
}

\author{
Rachna Kapoor $^{1}$ (D) James S. Welsh ${ }^{2,3} \cdot$ Vikas Dhawan $^{4} \cdot$ Seyed Alireza Javadinia ${ }^{5} \cdot$ Edward J. Calabrese $^{6}$. \\ Gaurav Dhawan ${ }^{7}$
}

Received: 11 June 2021 / Accepted: 20 July 2021 / Published online: 24 July 2021

(c) The Author(s), under exclusive licence to Springer-Verlag GmbH Germany, part of Springer Nature 2021

\begin{abstract}
Coronavirus variants are gaining strongholds throughout the globe. Despite early signals that SARS-CoV-2 coronavirus case numbers are easing up in the United States and during the middle of a (not so easy) vaccination roll out, the country has passed a grim landmark of 600,000 deaths. We contend that these numbers would have been much lower if the medical community undertook serious investigations into the potential of low doses of radiation (LDRT) as a mainstream treatment modality for COVID-19 pneumonia. LDRT has been posited to manifest anti-infectious and anti-inflammatory properties at doses of 0.3-1.0 Gy via the activation of the Nrf-2 pathway. Although some researchers are conducting well-designed clinical trials on the potential of LDRT, the deep-rooted, blind, and flawed acceptance of the Linear No-Threshold (LNT) model for ionizing radiation has led to sidelining of this promising therapy and thus unimaginable numbers of deaths in the United States.
\end{abstract}

\section{Introduction}

"It's never too late to drop your beliefs and let your wounds heal. Instead of wounding others as well"-Adam Scythe, Immortals, Vol. II. This quote fits aptly for the potential offered by low-dose radiation therapy (LDRT) in the treatment of COVID-19 and its newer and deadlier variants. The deep rooted and unfounded fear of low doses of radiation for the treatment of inflammatory and infectious diseases, including COVID-19, needs to be scientifically addressed

Rachna Kapoor

dr.rachnakapoor23@gmail.com

James S. Welsh

james.welsh@va.gov

Vikas Dhawan

vikasdhawan76@yahoo.co.in

Seyed Alireza Javadinia

Javadiniaa941@mums.ac.ir; Javadinia.alireza@gmail.com

Edward J. Calabrese

edwardc@schoolph.umass.edu

Gaurav Dhawan

drgdhawan@icloud.com

1 Saint Francis Hospital and Medical Center, Hartford, CT, USA immediately before millions of more precious human lives are lost.

\section{The SARS-CoV-2 coronavirus and its variants}

The SARS-CoV-2 RNA coronavirus that causes COVID19 has taken nearly 4 million human lives in just over a year (Johns Hopkins University \& Medicine, Coronavirus Resource Center 2021). The novel virus caught most countries off guard and ill-prepared to handle a pandemic of this

2 Edward Hines Jr VA Hospital, Hines, IL, USA

3 Stritch School of Medicine, Loyola University Chicago, Maywood, IL, USA

4 COVID 19 Facilities, Command Hospital (Western Command), Chandimandir, Panchkula, Haryana, India

5 Clinical Research Development Unit, Hospital Research Development Committee, Sabzevar University of Medical Sciences, Sabzevar, Iran

6 Department of Environmental Health Sciences, School of Public Health and Health Sciences, University of Massachusetts, Amherst, MA, USA

7 Sri Guru Ram Das University of Health Sciences, Amritsar, India 
scale and extent, resulting in widespread global devastation in terms of human mortality/morbidity and economy. Although several effective vaccines are now available, challenges have emerged with their distribution, storage/handling, administration, two-dose compliance, and the public's overall willingness to accept them. Some researchers have also raised doubts on the claims of $95 \%$ efficacy for these vaccines (Peter Doshi 2021). In addition, an unequal purchasing power of the high-income countries such as the European Union and the United States has pushed several low-income countries (e.g., in Africa) to the back of the queue for vaccine purchase and delivery, resulting in projected delays until late 2022 to cover their vulnerable populations (Tracking COVID-19 Vaccine Purchases across the globe 2021).

The sudden appearance and spread of SARS-CoV-2 variants, especially the UK variant (known as 20I/501Y.V1, VOC 202012/01, B.1.1.7 or the alpha variant) has been associated with increased transmissibility and mortality (What went wrong in the global governance of covid-19? 2021). Similarly, other variants have been identified such as the South African variant (known as 20H/501Y.V2, B.1.351 or the beta variant), Brazilian variant (known as P.1 or the gamma variant) and the Indian variants (e.g., B.1.617, the delta and kappa variants) which have the potential to either spread more easily, evade diagnostic tests, cause more severe forms of the disease, decrease susceptibility to some pharmacological agents or allow the virus to evade natural or vaccine induced immunity (Centers for Disease Control and Prevention -Science Brief: Emerging SARS-CoV-2 Variants $2021\}$.

\section{LDRT application in COVID-19—where do we stand?}

A key anti-viral and anti-inflammatory treatment that has been re-purposed for use against COVID-19 is low-dose radiation therapy (LDRT). LDRT has been recently resurrected and identified as a potentially life-saving treatment which was used prior to the "age of antibiotics" in the treatment of seriously ill patients with bacterial and/or viral pneumonia (Calabrese and Dhawan 2013a, b; Calabrese et al. 2014, 2015, 2019; Dhawan et al. 2019, 2020).

Table 1 summarizes the preliminary results of the clinical trials that evaluated the safety and efficacy of the LDRT in COVID-19 patients. Results from most of the published reports are encouraging and provide the preliminary evidence in this context; however, a recent randomized double blinded clinical trial on critically ill patients requiring mechanical ventilation, casted doubts on the efficacy of LDRT (Hess et al. 2021; Moreno-Olmedo et al. 2020; Sharma et al. 2020; Ameri et al. 2021; Sanmamed et al. 2021; Arenas et al. 2021; Papachristofilou et al. 2021). Several ongoing clinical trials continue to assess the safety and efficacy of LDRT in COVID-19 patients, and their results will shed additional light on the effectiveness and overall role of this treatment modality (The National Library of Medicine (NLM) 2020). In addition, these completed and ongoing clinical trials will help establish the optimal dose range as well as the timing of administration and the severity level of COVID-19 pneumonia, where LDRT is likely to be most effective.

\section{LDRT application-role of Nrf-2-timing is key}

Herein, we would like to propose that a single low dose of LDRT (0.3-1.0 Gy) has the potential for being effective against pneumonitis and acute respiratory distress syndrome (ARDS) induced by SARS-CoV2 and its emerging variants. The anticipated universal usefulness of LDRT in all such patients (irrespective of the specific causative SARS-CoV-2 variant or the myriad of clinical presentations) is linked to the induction of a potent antioxidant basic leucine zipper protein-Nrf2 (nuclear factor erythroid 2-related transcription factor). The Nrf2-induced antioxidant response drives immunological reactions toward an anti-inflammatory M2 phenotype. Activation of Nrf2 is dose dependent and displays features of a biphasic (hormetic) response (Calabrese et al. 2021).

Nrf2 is a transcription factor that activates immediate responses against oxidative, metabolic, and inflammatory stressors. Targeting Nrf2 is of prime importance and holds great promise in the treatment of many diseases typified by oxidative stress and inflammation, such as COVID-19-induced pneumonitis and ARDS.

In our opinion and as illustrated in Fig. 1, timing the administration of LDRT is extremely important for a successful patient outcome (Bevelacqua et al. 2021). We assert that there is a temporal window of opportunity during which LDRT can effectively address the respiratory symptoms of COVID-19 or other viral pneumonias. This temporal window concept is akin to the limited window of opportunity during which external beam radiation therapy can be effective for cancer-related spinal cord compression; if one waits too long, the window closes, and the clinical consequences become irreversible. In the case of COVID pneumonia, the optimal timing is before the patient becomes so ill that mechanical ventilation is required. In other words, LDRT and its consequential activation of Nrf2, is likely to work best before cytokine storm becomes overwhelming.

Nrf2 activation through LDRT will yield maximum patient benefits and offer the best chances of recovery if applied when cells have adequate energy to endure interactive, complementary, and corresponding adaptive (hormetic) immunological responses-in other words, prior to 


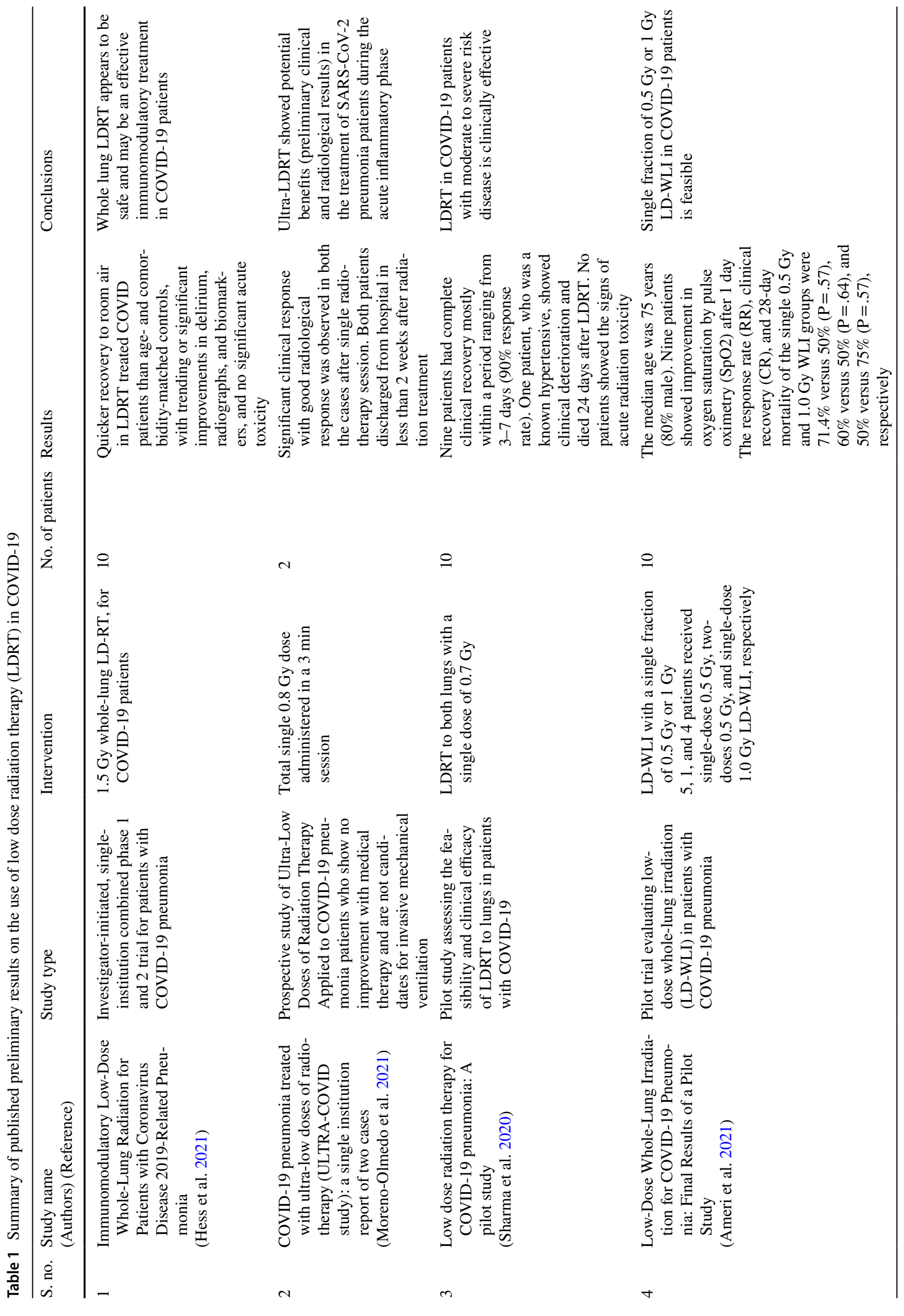




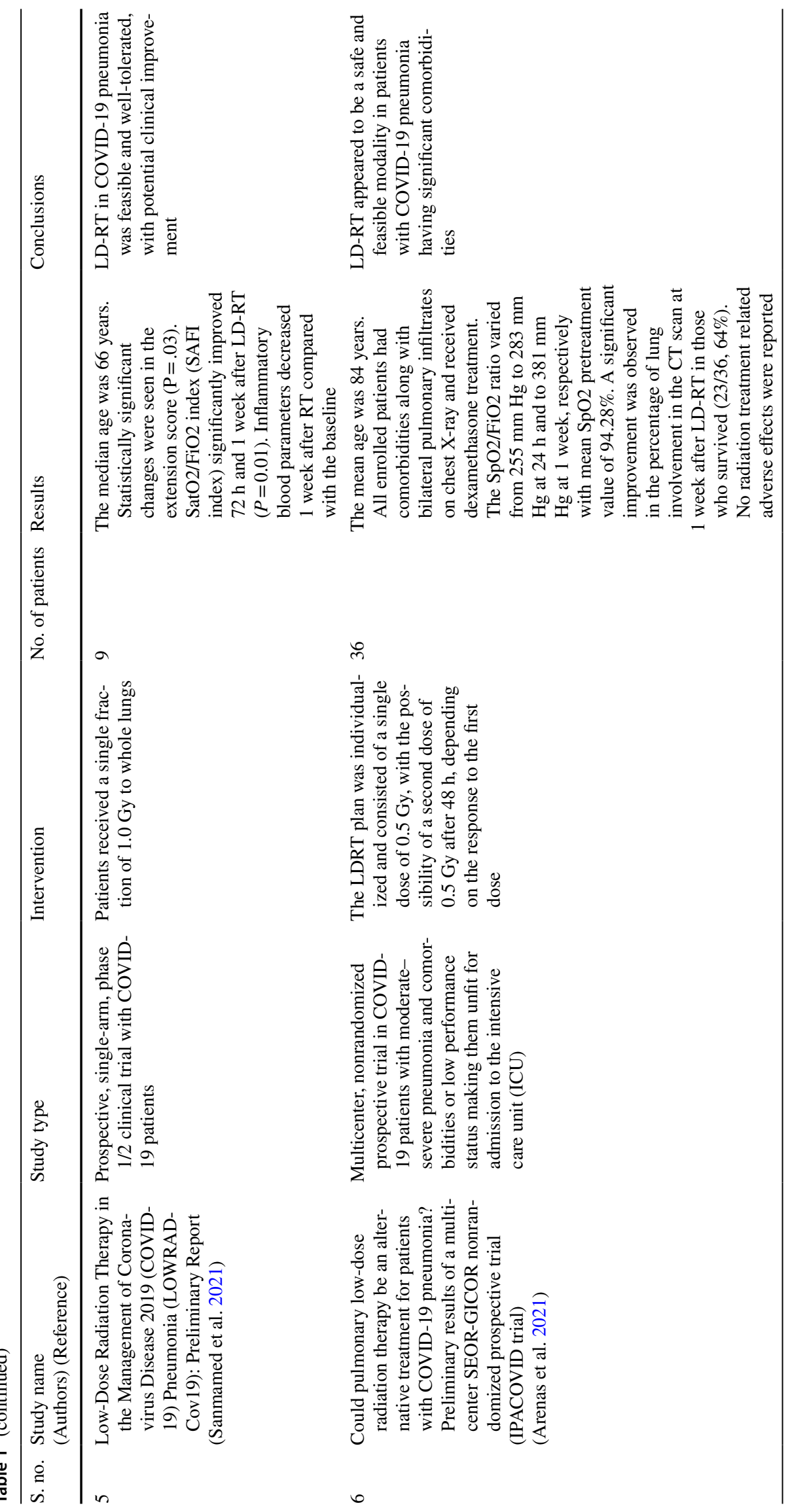




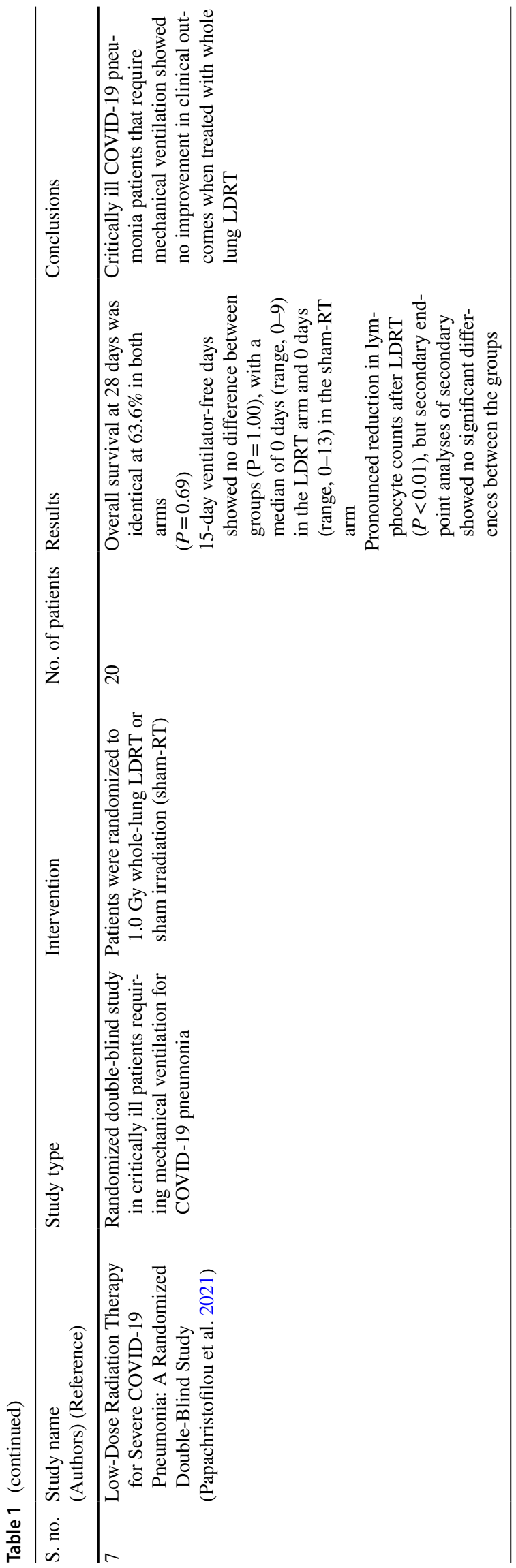

the occurrence of full-blown cytokine release syndrome. Chances of complete recovery from COVID-19 in patients on mechanical ventilators are small in all practical terms, even when LDRT is applied. Similarly, application of LDRT too early in the disease may prove counterproductive, especially if limited oxidative and controlled proinflammatory states are needed for successful generation of a fully effective immune response against the coronavirus.

\section{Death vs LDRT-easy choice-but it is complicated}

Despite several historical accounts of the benefits of LDRT in the treatment of inflammatory and infectious diseases, which have now been supplemented by encouraging results from ongoing clinical trials across the globe, the medical community still seems to be hesitant in widespread acceptance and application of this treatment.

One of the biggest concerns that has been raised by the medical fraternity is the risk of radiation-induced secondary malignancies (Dracham et al. 2018). In a recent study by Soyfer et al. (2021), the authors performed Monte Carlo calculations to assess the feasibility and potential harm of using low-dose $(0.75 \mathrm{~Gy})$ radiation to thoracic mid-plane of COVID-19 patients with moderate to severe disease using an orthovoltage machine. The authors concluded that using the data from the BEIR-VII committee of the US National Research Council, the potential benefits of delivering 0.75 Gy using an orthovoltage machine (minimum 300 $\mathrm{kVp}$ ) outweigh the likelihood of loss of life due to radiation-induced malignancy (Soyfer et al. 2021). Similarly in another simulation study, Roa et al. (2021) concluded that COVID-19 treatment with a single PA field prescription dose of 0.3 or 0.5 Gy to both lungs had a low likelihood of radiation-induced detriment to critical organs such as lungs, heart, spine, thymus, esophagus etc. The authors also stated that the benefit-to-risk ratio was very high for elderly patients who are likely to develop COVID-19-related complications and are unlikely to develop radiation-induced secondary malignancies (Roa et al. 2021).

Another linear no-threshold modeling-based analysis forecasted the risk of secondary cancers to be approximately $1 \%$ after 15-20 years with the administration of a single 0.5 Gy dose to the thorax (Schröder et al. 2019). Even if one accepts this likely overestimate, the risk of direct induced carcinogenic risk of LDRT (0.5-1.0 Gy) is still far below the risk of spontaneously occurring cancers (The 2007 recommendations of the international commission on radiological protection 2007). In a 2019 German study, researchers followed up 158 female patients treated with a cumulative average radiation therapy dose of $6.0 \mathrm{~Gy}$ for shoulderrelated non-malignant diseases and made comparisons 


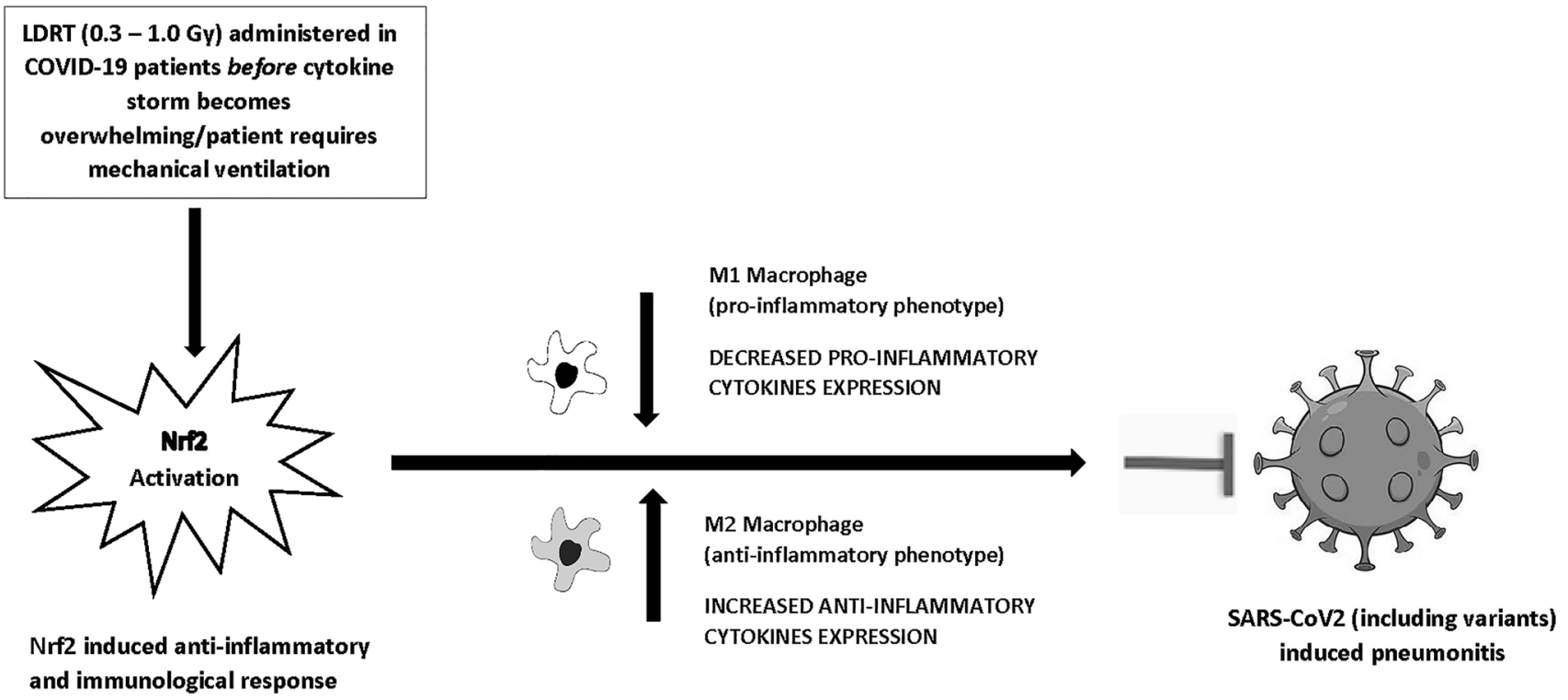

Fig. 1 LDRT induced Nrf2 activation and its effect on SARS-CoV-2 (including variants) induced pneumonitis (adapted from Calabrese et al. 2021)

with estimated spontaneous incidence of breast cancer in this cohort. After a median follow-up of over 21 years the researchers concluded that there were no increased rates of breast cancer for either the ipsilateral or the contralateral breasts (Zwicker et al. 2019).

The potential and real curative benefits of LDRT in the management of COVID-19 especially in patients with progressive and serious pneumonitis, outweighs these hypothetical cancer risks as a recent clinical trial has shown over $70 \%$ response rate with 0.5 Gy dosage (Ameri et al. 2021). Furthermore, the risk of pulmonary complications may be reduced by effective CT simulation treatment planning to detect and spare uninvolved lung parenchyma. This approach can reduce the irradiated volume of normal lung tissue, since diffuse pulmonary parenchymal involvement can be expected only in approximately half of COVID-19 patients (Ghahramani-Asl et al. 2020; Castelli et al. 2020). Other researchers have suggested quantifying the risk-benefit balance of LDRT in COVID-19 patients by considering background risk factors such as smoking and baseline heart disease risk to estimate radiation-induced lifetime risks of lung cancer and major coronary events (Shuryak et al. 2021). Therefore, considering the high mortality rates of severe COVID-19 (Olivas-Martínez et al. 2021) and the conceivable therapeutic role of LDRT, it seems reasonable to think again about its potential benefits and the number of lives that could be saved.

A recent study by Hanna et al. (2021), discussed some of the clinician-associated barriers to testing and using LDRT for COVID-19 lung disease in their practices. Not surprisingly, the perceived potential to do harm to the patient, including difficulties in properly predicting harm and the absence of hard data quantifying risks were amongst the biggest barriers (Hanna et al. 2021). The medical community generally has a very deep-rooted belief in the Linear NoThreshold (LNT) model, a concept that is flawed at its very origin but engrained profoundly in the minds of many due to its prolonged promulgation. Several recent articles have challenged this blind belief of the LNT model (Calabrese 2021a, 2021b; Siegel et al. 2018). We contend that unquestioned acceptance of LNT and the concomitant hesitancy to consider therapies based on low doses of radiation should be addressed immediately or millions of more lives, especially in the low-income countries having a serious lag in vaccine availability, may be unnecessarily lost.

Acknowledgements We thank all the frontline healthcare professionals who are fighting against COVID-19. E.J.C. acknowledges longtime support from the US Air Force (AFOSR FA9550-13-1-0047) and ExxonMobil Foundation (S18200000000256).

\section{Declarations}

Conflict of interest The authors declare that they have no conflict of interest.

\section{References}

Ameri A, Ameri P, Rahnama N, Mokhtari M, Sedaghat M, Hadavand F, Bozorgmehr R, Haghighi M, Taghizadeh-Hesary F (2021) Low-dose whole-lung irradiation for COVID-19 pneumonia: final 
results of a pilot study. Int J Radiat Oncol Biol Phys 109(4):859866. https://doi.org/10.1016/j.ijrobp.2020.11.065

Arenas M, Algara M, De Febrer G, Rubio C et al (2021) Could pulmonary low-dose radiation therapy be an alternative treatment for patients with COVID-19 pneumonia? Preliminary results of a multicenter SEOR-GICOR nonrandomized prospective trial (IPACOVID trial). Strahlenther Onkol 6:1-11. https://doi.org/10.1007/ s00066-021-01803-3

Bevelacqua JJ, Welsh JS, Mortazavi S (2021) Comments on: "Low dose radiation therapy for severe COVID-19 pneumonia: a randomized double-blind study." Int J Radiat Oncol Biol Phys S03603016(21):00445-00454. https://doi.org/10.1016/j.ijrobp.2021.04. 025

Calabrese EJ (2021a) LNT and cancer risk assessment: Its flawed foundations part 1: radiation and leukemia: where LNT began. Environ Res 18(197):111025. https://doi.org/10.1016/j.envres.2021.111025

Calabrese EJ (2021b) LNT and cancer risk assessment: Its flawed foundations part 2: How unsound LNT science became accepted. Environ Res 29(197):111041. https://doi.org/10.1016/j.envres.2021.111041

Calabrese EJ, Dhawan G (2013a) How radiotherapy was historically used to treat pneumonia: could it be useful today? Yale J Biol Med $86: 555-570$

Calabrese EJ, Dhawan G (2013b) The historical use radiotherapy in the treatment of sinus infection. Dose-Response 11:484-494

Calabrese EJ, Dhawan G, Kapoor R (2014) Use of X-rays to treat shoulder tendonitis/bursitis: a historical assessment. Arch Toxicol 88:1503-1517

Calabrese EJ, Dhawan G, Kapoor R (2015) The use of X-rays in the treatment of bronchial asthma: a historical assessment. Rad Res 184:180-192

Calabrese EJ, Dhawan G, Kapoor K, Kozumbo WJ (2019) Radiotherapy treatment of human inflammatory diseases and conditions: optimal dose. Hum Exp Toxicol 38(8):888-898

Calabrese EJ, Kozumbo WJ, Kapoor R, Dhawan G, Jimenez PCL, Giordano J (2021) NRF2 activation putatively mediates clinical benefits of low-dose radiotherapy in COVID-19 pneumonia and acute respiratory distress syndrome (ards): novel mechanistic considerations. Radiother Oncol 160:125-131. https://doi.org/10.1016/j. radonc. 2021.04.015

Castelli M, Maurin A, Bartoli A et al (2020) Prevalence and risk factors for lung involvement on low-dose chest CT (LDCT) in a paucisymptomatic population of 247 patients affected by COVID-19. Insights Imaging 11:117. https://doi.org/10.1186/s13244-020-00939-7

Centers for Disease Control and Prevention -Science Brief: Emerging SARS-CoV-2 Variants (2021) https://www.cdc.gov/coronavirus/ 2019-ncov/more/science-and-research/scientific-brief-emergingvariants.html

Dhawan G, Kapoor R, Dhamija A, Singh R, Monga B, Calabrese EJ (2019) Necrotizing fasciitis: low dose radiotherapy as a potential adjunct treatment. Dose-Response. https://doi.org/10.1177/15593 25819871757

Dhawan G, Kapoor R, Dhawan R, Dhawan G, Kapoor R, Calabrese EJ et al (2020) Low dose radiation therapy as a potential life saving treatment for COVID-19-induced acute respiratory distress syndrome(ARDS). Radiother Onocol 147:212-216. https://doi.org/ 10.1016/j.radonc.2020.05.002

Dracham CB, Shankar A, Madan R (2018) Radiation induced secondary malignancies: a review article. Radiat Oncol J 36(2):85-94. https:// doi.org/10.3857/roj.2018.00290

Ghahramani-Asl R, Porouhan P, Mehrpouyan M, Welsh JS, Calabrese EJ, Kapoor R, Dhawan G, Javadinia SA (2020) Feasibility of treatment planning system in localizing the COVID-19 pneumonia lesions and evaluation of volume indices of lung involvement. Dose Response 18(3):1559325820962600. https://doi.org/10.1177/1559325820 962600
Hanna CR, Robb KA, Blyth KG (2021) Clinician attitudes to using low dose radiotherapy to treat COVID-19 lung disease. Int J Radiat Oncol 109(4):886-890. https://doi.org/10.1016/j.jirobp.2020.12.003

Hess CB, Nasti TH, Dhere VR et al (2021) Immunomodulatory lowdose whole-lung radiation for patients with coronavirus disease 2019-related pneumonia. Int J Radiat Oncol Biol Phys 109(4):867879. https://doi.org/10.1016/j.ijrobp.2020.12.011

Johns Hopkins University \& Medicine (2021) Coronavirus Resource Center https://coronavirus.jhu.edu/

Moreno-Olmedo E, Suárez-Gironzini V, Pérez M et al (2021) COVID19 pneumonia treated with ultra-low doses of radiotherapy (ULTRA-COVID study): a single institution report of two cases. Strahlenther Onkol 197(5):429-437. https://doi.org/10.1007/ s00066-020-01743-4

Olivas-Martínez A, Cárdenas-Fragoso JL, Jiménez JV et al (2021) Inhospital mortality from severe COVID-19 in a tertiary care center in Mexico City; causes of death, risk factors and the impact of hospital saturation. PLoS ONE 16(2):e0245772. https://doi.org/10.1371/ journal.pone. 0245772

Papachristofilou A, Finazzi T, Blum A, Zehnder T, Zellweger N, Lustenberger J et al (2021) Low-dose radiation therapy for severe COVID19 pneumonia: a randomized double-blind study. Int J Radiat Oncol. https://doi.org/10.1016/j.ijrobp.2021.02.054

Peter Doshi: Pfizer and Moderna's "95\% effective" vaccines—we need more details and the raw data (2021) https://blogs.bmj.com/bmj/ 2021/01/04/peter-doshi-pfizer-and-modernas-95-effective-vaccineswe-need-more-details-and-the-raw-data/

Roa D, Moyses H, Leon S et al (2021) Dose simulations of an early 20th century kilovoltage pneumonia radiotherapy technique performed with a modern fluoroscope. Med Dosim 46(1):74-79. https://doi. org/10.1016/j.meddos.2020.08.002

Sanmamed N, Alcantara P, Cerezo E, Gaztañaga M, Cabello N, Gómez $S$ et al (2021) Low dose radiotherapy in the management of covid19 pneumonia (LOWRAD-Cov19) preliminary report. Int J Rad Oncol Biol Phys 109(4):880-885. https://doi.org/10.1016/j.ijrobp.2020.11. 049

Schröder A, Kriesen S, Hildebrandt G, Manda K (2019) First insights into the effect of low-dose X-Ray irradiation in adipose-derived stem cells. Int J Mol Sci 20(23):6075. https://doi.org/10.3390/ijms202360 75

Sharma DN, Guleria R, Wig N, Mohan A, Rath GK, Subramani V et al (2020) Low dose radiation therapy for COVID-19 pneumonia: a pilot study. medRxiv. https://doi.org/10.1101/2020.11.16.20231514

Shuryak I, Kachnic LA, Brenner DJ (2021) Lung cancer and heart disease risks associated with low-dose pulmonary radiotherapy to COVID19 patients with different background risks. Int J Radiat Oncol Biol Phys. https://doi.org/10.1016/j.ijrobp.2021.04.018

Siegel JA, Pennington CW, Sacks B, Welsh JS (2018) The birth of the illegitimate linear no-threshold model. Am J Clin Oncol 41(2):173177. https://doi.org/10.1097/COC.0000000000000244

Soyfer V, Socol Y, Bragilovski D, Corn BW (2021) The theoretical value of whole-lung irradiation for COVID-19 pneumonia: a reasonable and safe solution until targeted treatments are developed. Radiat Res. https://doi.org/10.1667/RADE-20-00261.1

The National Library of Medicine (NLM) (2021) Clinical Trials Gov. https://clinicaltrials.gov/ct2/results?cond=COVID\&term $=$ low + dose + radiation $\&$ cntry $=\&$ state $=\&$ city $=\&$ dist $=$. Accessed 26 June 2021

The 2007 recommendations of the international commission on radiological protection (2007) ICRP publication 103. Ann ICRP 37(2-4): 1332. https://doi.org/10.1016/j.icrp.2007.10.003

Tracking COVID-19 Vaccine Purchases across the globe (2021) https:// launchandscalefaster.org/covid-19/vaccinepurchases

What went wrong in the global governance of covid-19? (2021) https:// www.bmj.com/content/372/bmj.n303 
Zwicker F, Kirchner C, Huber PE, Debus J, Zwicker H, Klepper R (2019)

Breast cancer occurrence after low dose radiotherapy of nonmalignant disorders of the shoulder. Sci Rep 9(1):5301. https://doi.org/10. 1038/s41598-019-41725-w

Publisher's Note Springer Nature remains neutral with regard to jurisdictional claims in published maps and institutional affiliations. 